\title{
Guggulsterone inhibits melanogenesis in B16 murine melanoma cells by downregulating tyrosinase expression
}

\author{
JEUNG-HYUN KOO $^{1 *}$, KYOUNG-SUK RHEE ${ }^{2 *}$, HYOUNG-WON KOH $^{1}$, HYUN-YOUNG JANG $^{1}$, \\ BYUNG-HYUN PARK ${ }^{1,3,4}$ and JIN-WOO PARK ${ }^{1}$ \\ Departments of ${ }^{1}$ Biochemistry and ${ }^{2}$ Internal Medicine; ${ }^{3}$ Research Institute for Endocrine Sciences; \\ ${ }^{4}$ Diabetes Research Center, Chonbuk National University Medical School, Jeonju, Jeonbuk 561-756, Republic of Korea
}

Received April 10, 2012; Accepted June 19, 2012

DOI: $10.3892 /$ ijmm.2012.1057

\begin{abstract}
In the present study, we investigated the effect of guggulsterone on melanogenesis in B16 melanoma cells and elucidated its possible mechanism of action. The effects of guggulsterone on melanogenesis were determined by assaying melanin synthesis and cellular tyrosinase activity in B16/ F10 mouse melanoma cells. Guggulsterone dose-dependently inhibited isobutylmethylxanthine (IBMX)-induced melanogenesis and cellular tyrosinase activity with no cytotoxicity. Decreased melanin biosynthesis was accompanied by the reduced expression of melanogenesis-related genes, such as tyrosinase, microphthalmia-associated transcription factor, tyrosinase-related protein (TRP)-1 and TRP-2. Guggulsterone also inhibited $\alpha$-melanocyte stimulating hormone- or forskolininduced increases in melanogenesis, suggesting an action on the cAMP-dependent melanogenic pathway. Co-incubation with chenodeoxycholic acid, a well-known farnesoid-X receptor agonist, did not affect IBMX-induced melanogenesis. These results suggest that guggulsterone exerts a melanogenic inhibitory effect through the downregulation of tyrosinase expression.
\end{abstract}

\section{Introduction}

Melanin is synthesized in the melanosomes of melanocytes by a process known as melanogenesis and plays a crucial role in protecting the skin from the harmful effects of ultraviolet (UV) radiation and diverse free radicals. Melanogenesis is regulated by at least three melanogenic enzymes, tyrosinase, tyrosinase-related protein (TRP)-1 and TRP-2 (1). Tyrosinase

Correspondence to: Dr Byung-Hyun Park or Dr Jin-Woo Park, Department of Biochemistry, Chonbuk National University Medical School, 567 Baekje-daero, Deokjin-gu, Jeonju, Jeonbuk 561-756, Republic of Korea

E-mail: bhpark@jbnu.ac.kr

E-mail: jinwoo@jbnu.ac.kr

${ }^{*}$ Contributed equally

Key words: guggulsterone, melanogenesis, tyrosinase, cyclic AMP is a rate-limiting enzyme that catalyzes the first two steps in the melanin biosynthetic pathway: hydroxylation of tyrosine to 3,4-dihydroxyphenylalanine (DOPA) and oxidation of DOPA to DOPAquinone (2). TRP-2, which functions as a DOPAchrome tautomerase, catalyzes the rearrangement of DOPAchrome to 5,6-dihydroxyindole-2-carboxylic acid (DHICA) (3), and TRP-1 oxidizes DHICA to a carboxylated indole-quinone (4).

Understanding the regulation of melanogenesis is of great interest pharmaceutically and cosmeceutically as melanogenesis inhibitors can be used for the treatment of hyper-pigmentationrelated diseases, such as melasma, lentigines, nevus, ephelis, freckles and age spots (5). Of the various signaling pathways that regulate melanogenesis, the cyclic AMP (cAMP)-dependent signaling pathway plays a pivotal role. cAMP-elevating agents, such as $\alpha$-melanocyte stimulating hormone ( $\alpha-\mathrm{MSH})$, isobutylmethylxanthine (IBMX) and forskolin stimulate melanogenic processes in the human epidermis $(6,7)$. cAMP increases the expression of melanogenic enzymes partly through protein kinase A (PKA). PKA phosphorylates the cAMP responsive element binding protein (CREB), which induces the expression of microphthalmia-associated transcription factor (MITF) (8). MITF is known as a master regulator of melanocyte development, survival, differentiation and melanogenesis (9). It also regulates the transcription of three major melanogenic enzymes: tyrosinase, TRP-1 and TRP-2.

Flavonoids are a group of polyphenolic compounds widely distributed in plants. Their potent bioactivity and relatively low toxicity have rendered them attractive for use as active ingredients in functional foods and cosmetics. Guggulsterone [4,17(20)-pregnadiene-3,16-dione], which is the active component of gugulipid, is derived from the gum resin (guggulu) of the tree, Commiphora mukul. This gum resin has been used for centuries in Ayurvedic medicine to treat obesity, arthritis and hyperlipidemia $(10,11)$. In addition, guggulsterone has been reported to act as a farnesoid X receptor (FXR) antagonist $(12,13)$. Therefore, it can effectively regulate bile acid synthesis (11-14) and carbohydrate metabolism (15). We have previously studied the effects of guggulsterone on type 1 diabetes (16) and arthritis (17). However, to our knowledge, there is no report on effect of guggulsterone on melanogenesis. During screening for new melanogenesis-inhibiting agents from flavonoids, we found that guggulsterone effectively inhibited melanogenesis. Therefore, in this study, we investigated the 
inhibitory mechanism of guggulsterone against IBMX-induced melanogenesis in B16 melanoma cells.

\section{Materials and methods}

Cells and materials. The B16/F10 mouse melanoma cell line was obtained from the Korean Cell Line Bank (Seoul, Korea). Cells were cultured in DMEM containing $10 \%$ fetal bovine serum, $100 \mathrm{U} / \mathrm{ml}$ penicillin, $0.1 \mathrm{mg} / \mathrm{ml}$ streptomycin, and $0.25 \mu \mathrm{g} / \mathrm{ml}$ amphotericin $\mathrm{B}$ at $37^{\circ} \mathrm{C}$ in a humidified $95 \%$ air $/ 5 \%$ $\mathrm{CO}_{2}$ atmosphere. Guggulsterone was obtained from Alexis Biochemicals (Lausen, Switzerland), and 6-ethyl chenodeoxycholic acid (CDCA), $\alpha-\mathrm{MSH}$, IBMX, and forskolin were obtained from Sigma (St. Louis, MO, USA). Drug treatment began $24 \mathrm{~h}$ after seeding, and cells were harvested after two days of incubation.

Melanin content measurement. The melanin contents of the cultured B16 cells were measured as described previously (18). The cells were washed twice with phosphate-buffered saline (PBS) and lysed with $20 \mathrm{mM}$ Tris-0.1\% Triton X-100 (pH 7.5). Cell lysates were precipitated with the same amount of $20 \%$ trichloroacetic acid. After washing twice with $10 \%$ trichloroacetic acid, the pellets were treated with ethyl alcohol:diethyl ether (3:1) and diethyl ether in succession. The samples were air-dried, dissolved in $1 \mathrm{ml}$ of $0.85 \mathrm{M} \mathrm{KOH}$, and boiled for $15 \mathrm{~min}$. After cooling, absorbance was measured with a spectrophotometer at $440 \mathrm{~nm}$. The amount of cellular melanin was corrected according to the DNA content of the samples. The DNA content was determined using the fluorescence assay of bisbenzimide H 33258 using a DNA Quantification kit (Sigma).

Tyrosinase activity assay. Tyrosinase activity was assayed as DOPA oxidase activity with some modifications, as described previously (18). Briefly, cell lysate was obtained after washing twice with PBS. Tyrosinase activity was then analyzed spectrophotometrically by following the oxidation of DOPA to DOPAchrome at $475 \mathrm{~nm}$. The reaction mixture containing $100 \mu \mathrm{l}$ of freshly prepared substrate solution $(0.1 \% \mathrm{~L}-\mathrm{DOPA}$ in $0.1 \mathrm{M}$ sodium phosphate, $\mathrm{pH}$ 6.0) and $50 \mu \mathrm{l}$ of enzyme solution was incubated at $37^{\circ} \mathrm{C}$. The absorbance change was measured during the first $10 \mathrm{~min}$ of the reaction, while the increase of the absorbance was linear. Corrections for the auto-oxidation of L-DOPA in the controls were made. The tyrosinase activity was corrected according to the DNA content of the samples and presented as a percentage of IBMX-treated control cells.

MTT assay. The viability of the cultured cells was determined by the reduction of 3-(4,5-dimethylthiazol-2-yl)-2,5-diphenyltetrazolium bromide (MTT) to formazan. The cells were seeded in 96-well plates and cultured for $24 \mathrm{~h}$. Following drug treatment, MTT $(5 \mathrm{mg} / \mathrm{ml}$ in PBS, $100 \mu \mathrm{l})$ was added to each well. The cells were incubated at $37^{\circ} \mathrm{C}$ for $30 \mathrm{~min}$, and dimethyl sulfoxide $(100 \mu \mathrm{l})$ was then added to dissolve the formazan crystals. The absorbance was measured at $570 \mathrm{~nm}$ with a spectrophotometer.

Western blot analysis. Cells were homogenized in ice-cold lysis buffer. The homogenates containing $10 \mu \mathrm{g}$ of protein were separated by SDS-PAGE with $10 \%$ resolving and $3 \%$ acrylamide stacking gel and transferred to a nitrocellulose membrane in a western blot analysis apparatus run at $100 \mathrm{~V}$ for $1.5 \mathrm{~h}$. The nitrocellulose membrane was blocked with $2 \%$ bovine serum albumin and then incubated overnight with $1 \mu \mathrm{g} / \mathrm{ml}$ goat anti-murine tyrosinase IgG (Santa Cruz Biotechnology, Inc., Santa Cruz, CA, USA). The binding of the antibody was detected with anti-goat IgG conjugated with horseradish peroxidase (Sigma). Immunoblots were developed using an Enhanced Chemiluminescence Plus kit (Amersham Biosciences, Buckinghamshire, UK), and the intensity of the bands was measured by LAS-1000 (Fujifilm, Tokyo, Japan).

Real-time RT-PCR. Total RNA was prepared from the cells using TRIzol reagent (Invitrogen, Carlsbad, CA, USA). Total RNA $(2 \mu \mathrm{g})$ was treated with RNase-free DNase (Invitrogen), and first-strand cDNA was generated using random hexamer primers provided in the first-strand cDNA synthesis kit (Applied Biosystems, Foster City, CA, USA). Specific primers for each gene (Table I) were designed using Primer Express software (Applied Biosystems). The real-time RT-PCR reaction mixture consisted of $10 \mathrm{ng}$ reverse transcribed total RNA, $167 \mathrm{nM}$ forward and reverse primers, and 2X PCR master mixture in a final volume of $10 \mu \mathrm{l}$. PCR reactions were carried out in 384-well plates using the ABI PRISM 7900HT Sequence Detection System (Applied Biosystems). All the experiments were performed in triplicate.

Statistical analysis. Statistical analysis of the data was performed using ANOVA and Duncan's test. $\mathrm{P}<0.05$ was considered to indicated statistically significant differences.

\section{Results}

Guggulsterone inhibits melanogenesis in B16 cells. In order to investigate the effects of guggulsterone on IBMX-induced melanogenesis, the melanin contents in the B16 cells were measured following treatment with guggulsterone. At concentrations of $1,5,10$, and $25 \mu \mathrm{M}$ guggulsterone, melanin production was compared with the untreated controls (Fig. 1). At concentrations of $1,5,10$, and $25 \mu \mathrm{M}$, guggulsterone decreased melanin production to $104.5 \pm 5.0,54.1 \pm 5.8,39.4 \pm 4.8$ and $33.8 \pm 3.4 \%$, respectively in a dose-dependent manner without obvious cytotoxicity at any of the concentrations tested.

Guggulsterone decreases the expression of melanogenesisrelated genes. Since tyrosinase is the rate-limiting enzyme for melanin biosynthesis, the effect of guggulsterone on tyrosinase activity was determined. Cellular tyrosinase activity was decreased by guggulsterone in a dose-dependent manner (Fig. 2), which was consistent with the decreased melanin content (Fig. 1). The expression of tyrosinase protein was determined by western blot analysis (Fig. 3). The results showed that the tyrosinase protein was greatly increased by IBMX treatment, and this induction was significantly inhibited by guggulsterone in a dose-dependent manner. The expression of tyrosinase mRNA determined by real-time RT-PCR also exhibited a significant decrease following guggulsterone treatment (Fig. 4). These results indicated that the inhibition of tyrosinase by guggulsterone was exerted at the transcriptional level. The 
Table I. Sequences and accession numbers for forward (F) and reverse (R) primers used in real-time RT-PCR.

\begin{tabular}{lll}
\hline Gene & Sequences for primers & Accession no. \\
\hline Tyrosinase & F, TTGCCACTTCATGTCATCATAGAATATT & NM011661 \\
TRP1 & R, TTTATCAAAGGTGTGACTGCTATACAAAT & NM031202 \\
FRP2 & R, CGTTGTTCCAACGGGAAGGT & \\
& F, CTCAGAGCTCGGGCTCAGTT & X63349 \\
MITF & R, TGTTCAGCACGCCATCCA & NM008601 \\
FAPDH & R, CGCCTGATCTGGTGAATCG & NM008084 \\
& F, CGTCCCGTAGACAAAATGGT & \\
\hline
\end{tabular}

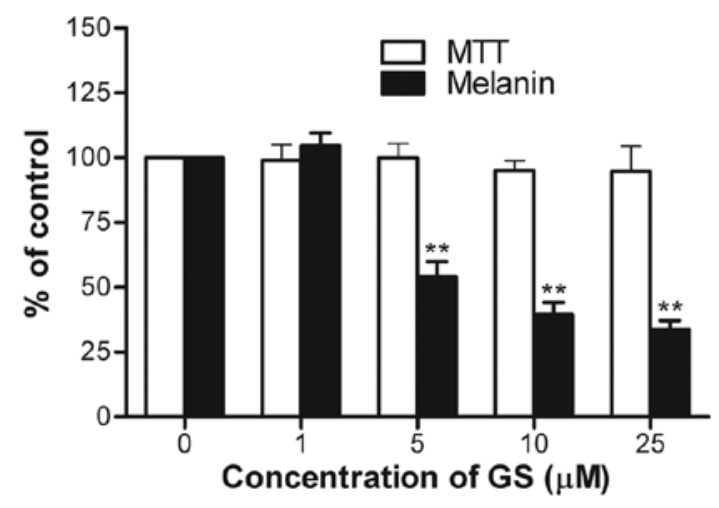

Figure 1. Effect of guggulsterone on melanin content in B16 melanoma cells. Cells $\left(5 \times 10^{6}\right.$ cells $/$ well $)$ were incubated with various concentrations of guggulsterone in the presence of $0.1 \mathrm{mM}$ IBMX for two days. Melanin content was determined as described in 'Materials and methods'. Cell viability was determined by the MTT assay. Data are expressed as a percentage of the IBMXtreated control cells and presented as the means $\pm \operatorname{SEM}(n=11) .{ }^{* *} \mathrm{P}<0.01$ vs. untreated control. GS, guggulsterone.

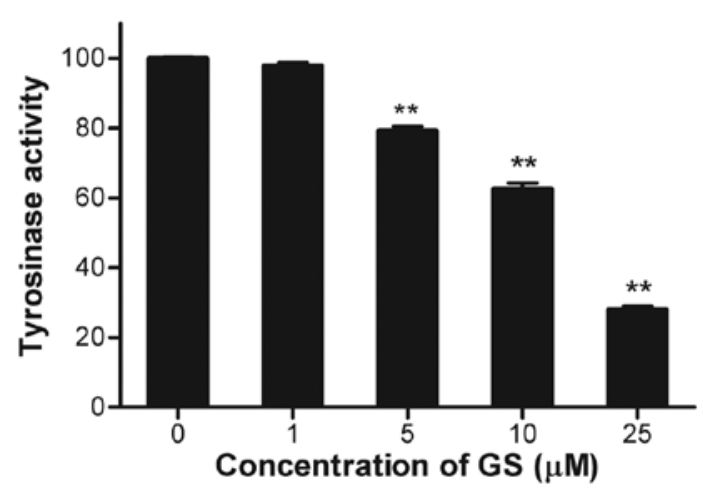

Figure 2. Effect of guggulsterone on cellular tyrosinase activity. Cells $\left(5 \times 10^{6}\right.$ cells) were treated with various concentrations of guggulsterone in the presence of $0.1 \mathrm{mM}$ IBMX for two days. Tyrosinase activity in cellular lysate was determined. Data are expressed as a percentage of the IBMX-treated controls and presented as the mean $\pm \operatorname{SEM}(n=9)$. ${ }^{* *} \mathrm{P}<0.01$ vs. untreated control. GS, guggulsterone.

mRNA levels of TRP-1, TRP-2 and MITF, members of the melanogenesis-related gene family, were also decreased by the presence of guggulsterone (Fig. 4).

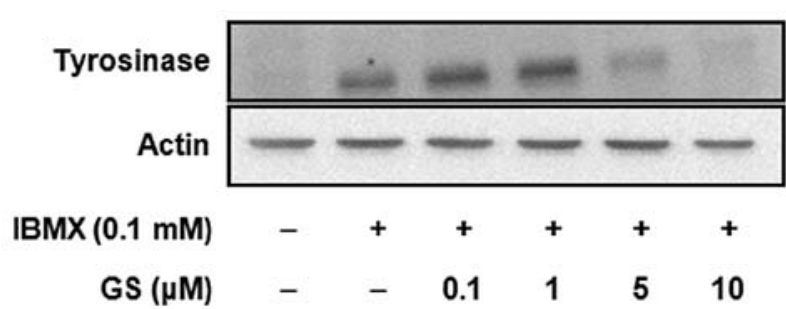

Figure 3. Effect of guggulsterone on tyrosinase protein expression. Cells $\left(5 \times 10^{6}\right.$ cells) were treated with a range of concentrations (1-25 $\left.\mu \mathrm{M}\right)$ of guggulsterone in the presence or absence of $0.1 \mathrm{mM}$ IBMX for two days. Tyrosinase protein levels were analyzed by western blot analysis. Experiments were performed three times with similar results, and a typical result was presented. GS, guggulsterone.

Guggulsterone inhibits cAMP-elevating agent-induced melanogenesis. When the B16 cells were incubated with IBMX, the cell suspension turned black, indicating increased cellular melanogenesis (Fig. 5). The cellular melanin contents were also markedly increased in the cells treated with $5 \mu \mathrm{M} \alpha-\mathrm{MSH}$ or $5 \mu \mathrm{M}$ forskolin. However, the presence of guggulsterone significantly inhibited melanogenesis induced by both $\alpha-\mathrm{MSH}$ and forskolin (Fig. 5), suggesting that guggulsterone regulates melanogenesis through the cAMP-dependent pathway.

The inhibitory effect of guggulsterone on IBMX-induced melanogenesis was not affected when the B16 cells were treated with both guggulsterone and CDCA, an antagonist and an agonist for FXR, respectively (Fig. 6). These results indicated that the effect of guggulsterone on melanogenesis was not mediated by antagonizing the FXR signaling pathway. Again, treatment with guggulsterone or CDCA alone did not affect melanogenesis at the concentration used in this study.

\section{Discussion}

We performed this study to examine whether guggulsterone can be used as a whitening cosmetic agent. To answer this question, we first evaluated whether guggulsterone can inhibit melanogenesis in IBMX-treated B16 melanoma cells. When the B16 cells were treated with guggulsterone, a dose-dependent inhibition of melanin production was observed. This result cannot be explained by the cytotoxicity of guggulsterone, as 
Tyrosinase

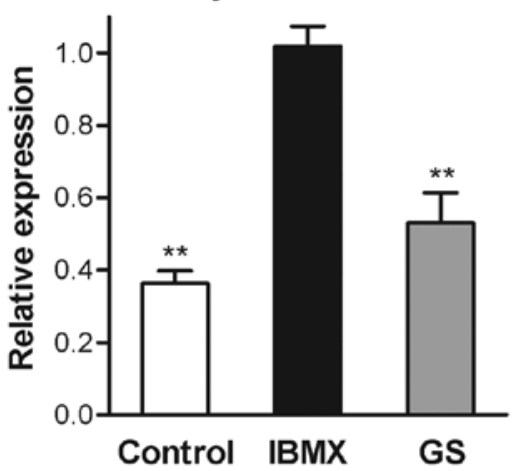

TRP-2

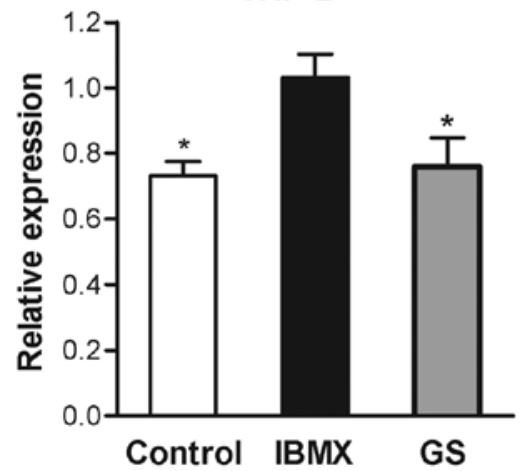

TRP-1
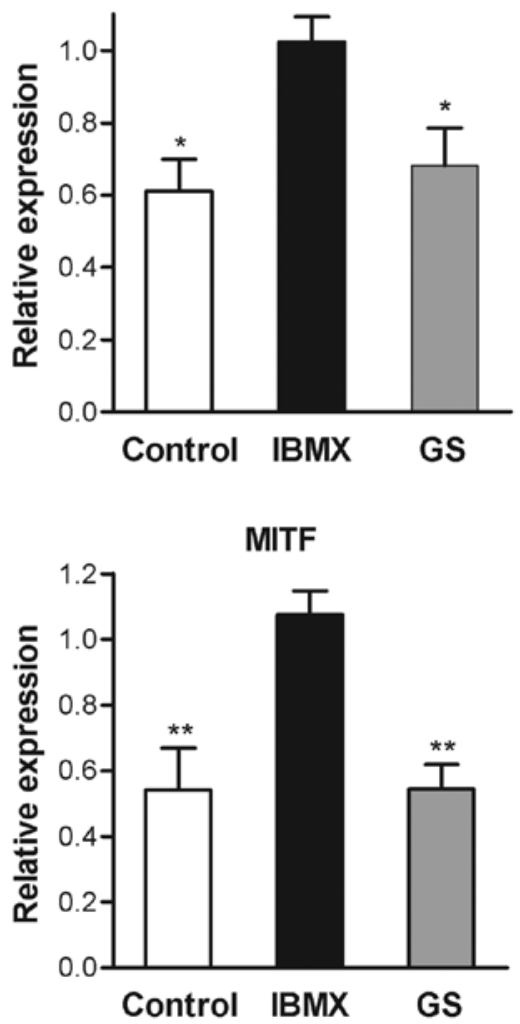

Figure 4. Effect of guggulsterone on the expression of melanogenesis-related genes. Cells (5x10 ${ }^{6}$ cells) were treated with 0.1 mM IBMX for two days in the presence or absence of $10 \mu \mathrm{M}$ guggulsterone. The cells were then harvested, and total RNA was extracted. mRNA expression was quantified by real-time RT-PCR and normalized to GAPDH. Data are expressed as a relative expression of IBMX-treated cells and presented as the means \pm SEM ( $=8$ ). ${ }^{*}<0.05$; ${ }^{* *} \mathrm{P}<0.01$ vs. IBMX-treated control. GS, guggulsterone.

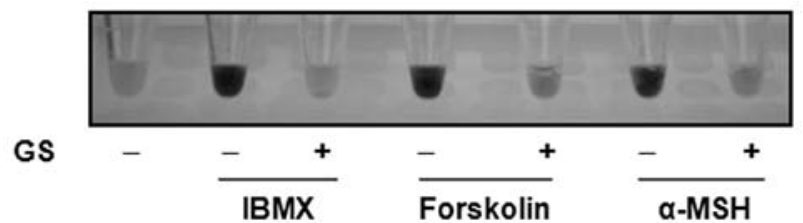

Figure 5. Effect of guggulsterone on $\alpha$-MSH-, IBMX- and forskolin-induced melanogenesis. Cells $\left(5 \times 10^{6}\right)$ were incubated with $10 \mu \mathrm{M}$ guggulsterone in the presence of IBMX $(0.1 \mathrm{mM}), \alpha-\mathrm{MSH}(5 \mu \mathrm{M})$, or forskolin $(5 \mu \mathrm{M})$ for two days, collected in a microfuge tube, and photographed. Experiments were performed three times with similar results, and a typical photograph was presented. GS, guggulsterone.

there was no evident decrease in the number of viable cells up to a concentration of $25 \mu \mathrm{M}$.

UV-induced hyperpigmentation occurs in two stages, an immediate darkening and a delayed tanning reaction. Immediate pigment darkening is thought to result from the oxidation of pre-existing melanin and redistribution of melanosomes. By contrast, the delayed tanning response that is photoprotective against subsequent UV injury begins as the immediate pigmentation reaction fades and progresses for at least three to five days after UV exposure (19). Delayed tanning is preceded by the increase in tyrosinase activity in melanocytes $(5,19)$. Since tyrosinase catalyzes the rate-limiting reaction of the melanogenic process, any reduction in the amount of enzyme activity or expression will result in a corresponding decrease in the amount of melanin synthesized. Indeed, in B16 cells treated

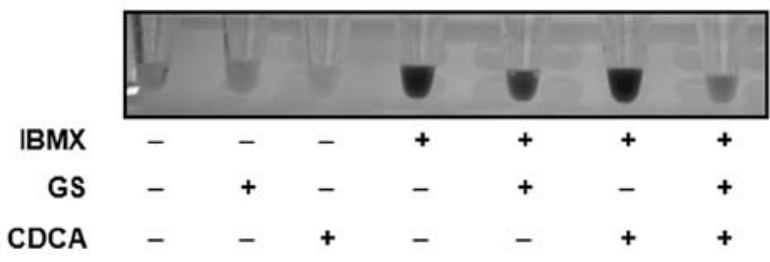

Figure 6. Effects of guggulsterone and chenodeoxycholic acid (CDCA) on IBMX-induced melanogenesis. Cells $\left(5 \times 10^{6}\right)$ were incubated with $10 \mu \mathrm{M}$ guggulsterone or $10 \mu \mathrm{M}$ CDCA in the presence or absence of IBMX $(0.1 \mathrm{mM})$ for two days, collected in a microfuge tube, and photographed. Experiments were performed three times with similar results, and a typical photograph was presented. GS, guggulsterone.

with IBMX, there were marked increases in tyrosinase activity, namely increases in protein and mRNA expression, which were similar to those of the delayed tanning response after UV irradiation. Accordingly, the treatment of cells with guggulsterone resulted in dose-dependent inhibition of the enzymatic activity and expression of tyrosinase. These results indicate that guggulsterone inhibits IBMX-induced melanogenesis in B16 cells through the suppression of tyrosinase expression.

The melanocyte-keratinocyte complex of the skin responds quickly to a wide range of environmental stimuli, often through paracrine and/or autocrine means. IBMX is known to increase cellular cAMP through the inhibition of the cAMP-degrading enzyme, phosphodiesterase (20). Guggulsterone effectively blocked the IBMX-induced increase in melanogenesis by 
decreasing the expression of tyrosinase. This effect occurred at the transcriptional level, suggesting its action on the cAMPdependent pathway. When the B16 cells were treated with $\alpha-\mathrm{MSH}$, a peptide acting on melanocortin 1-receptor (MC1-R) of melanocytes (21), or forskolin, a direct activator of adenylate cyclase (22), the cellular melanin contents were significantly increased. Again, guggulsterone significantly inhibited melanogenesis induced by both $\alpha-\mathrm{MSH}$ and forskolin, as in the case of IBMX stimulation. These results also support the action mechanism of guggulsterone on the cAMP-dependent pathway. In addition to the cAMP/PKA pathway, increased melanogenesis after UV irradiation was thought to occur through the activation of the diacylglycerol/protein kinase $\mathrm{C}$ (PKC) and nitric oxide/protein kinase $\mathrm{G}$ (PKG) pathways, and SOS response to UV-induced DNA damage (5). The PKC-induced activation of tyrosinase occurs through phosphorylation rather than the synthesis of new enzymes (23). However, PKG is known to increase the expression of tyrosinase protein (24). The additional effects of guggulsterone on these pathways require further study.

Safety following long-term application is a very important issue for therapeutic compounds. In recent years, naturally occurring herbal extracts and flavonoids have gained attention as putative hypopigmenting agents $(18,25-27)$. In the case of guggulsterone, no toxicity was observed after oral administration $(75 \mathrm{mg} / \mathrm{kg}$ ) for eight weeks in laboratory rats (28). The topical application of guggulsterone prior to 12-O-tetradecanoylphorbol-13-acetate (TPA) application onto mouse skin resulted in a significant inhibition against TPA-induced skin edema and hyperplasia without any noticeable side-effects (29). In addition, guggulsterone has long been used in traditional medicine. This evidence suggests the possibility of guggulsterone as a safe hypopigmenting agent.

In conclusion, to our knowledge, the present study demonstrates for the first time that guggulsterone is an effective inhibitor of tyrosinase and inhibits melanin biosynthesis. Even though we have not determined its effects in in vivo conditions, guggulsterone may have beneficial effects in the treatment of hyperpigmentation diseases.

\section{Acknowledgements}

The present study was supported by a National Research Foundation of Korea grant funded by the Korean Government (no. 2011-0028222).

\section{References}

1. Yamaguchi $Y$ and Hearing VJ: Physiological factors that regulate skin pigmentation. Biofactors 35: 193-199, 2009.

2. Hearing VJ and Jimenez M: Mammalian tyrosinase - the critical regulatory control point in melanocyte pigmentation. Int J Biochem 19: 1141-1147, 1987.

3. Yokoyama K, Yasumoto K, Suzuki H and Shibahara S: Cloning of the human DOPAchrome tautomerase/tyrosinase-related protein 2 gene and identification of two regulatory regions required for its pigment cell-specific expression. J Biol Chem 269: 27080-27087, 1994.

4. Kobayashi T, Urabe K, Winder A, et al: Tyrosinase related protein 1 (TRP1) functions as a DHICA oxidase in melanin biosynthesis. EMBO J 13: 5818-5825, 1994.

5. Costin GE and Hearing VJ: Human skin pigmentation: melanocytes modulate skin color in response to stress. FASEB J 21: 976-994, 2007.
6. Busca R and Ballotti R: Cyclic AMP a key messenger in the regulation of skin pigmentation. Pigment Cell Res 13: 60-69, 2000.

7. Im S, Moro O, Peng F, et al: Activation of the cyclic AMP pathway by alpha-melanotropin mediates the response of human melanocytes to ultraviolet B radiation. Cancer Res 58: 47-54, 1998.

8. Bertolotto C, Abbe P, Hemesath TJ, et al: Microphthalmia gene product as a signal transducer in cAMP-induced differentiation of melanocytes. J Cell Biol 142: 827-835, 1998.

9. Wan P, Hu Y and He L: Regulation of melanocyte pivotal transcription factor MITF by some other transcription factors. Mol Cell Biochem 354: 241-246, 2011.

10. Sinal CJ and Gonzalez FJ: Guggulsterone: an old approach to a new problem. Trends Endocrinol Metab 13: 275-276, 2002.

11. Urizar NL and Moore DD: GUGULIPID: a natural cholesterollowering agent. Annu Rev Nutr 23: 303-313, 2003.

12. Urizar NL, Liverman AB, Dodds DT, et al: A natural product that lowers cholesterol as an antagonist ligand for FXR. Science 296: 1703-1706, 2002.

13. Wu J, Xia C, Meier J, Li S, Hu X and Lala DS: The hypolipidemic natural product guggulsterone acts as an antagonist of the bile acid receptor. Mol Endocrinol 16: 1590-1597, 2002.

14. Cui J, Huang L, Zhao A, et al: Guggulsterone is a farnesoid X receptor antagonist in coactivator association assays but acts to enhance transcription of bile salt export pump. J Biol Chem 278: 10214-10220, 2003.

15. Stayrook KR, Bramlett KS, Savkur RS, et al: Regulation of carbohydrate metabolism by the farnesoid X receptor. Endocrinology 146: 984-991, 2005.

16. Lv N, Song MY, Kim EK, Park JW, Kwon KB and Park BH: Guggulsterone, a plant sterol, inhibits NF- $\kappa \mathrm{B}$ activation and protects pancreatic beta cells from cytokine toxicity. Mol Cell Endocrinol 289: 49-59, 2008.

17. Lee YR, Lee JH, Noh EM, et al: Guggulsterone blocks IL-1 $\beta$ mediated inflammatory responses by suppressing NF- $\kappa \mathrm{B}$ activation in fibroblast-like synoviocytes. Life Sci 82: 1203-1209, 2008.

18. Koo JH, Lee I, Yun SK, Kim HU, Park BH and Park JW: Saponified evening primrose oil reduces melanogenesis in B16 melanoma cells and reduces UV-induced skin pigmentation in humans. Lipids 45: 401-407, 2010.

19. Eller MS and Gilchrest BA: Tanning as part of the eukaryotic SOS response. Pigment Cell Res 13 (Suppl 8): S94-S97, 2000.

20. Beavo JA, Rogers NL, Crofford OB, Hardman JG, Sutherland EW and Newman EV: Effects of xanthine derivatives on lipolysis and on adenosine 3',5'-monophosphate phosphodiesterase activity. Mol Pharmacol 6: 597-603, 1970.

21. Wakamatsu K, Graham A, Cook D and Thody AJ: Characterisation of ACTH peptides in human skin and their activation of the melanocortin-1 receptor. Pigment Cell Res 10: 288-297, 1997.

22. Tamagawa T, Niki H and Niki A: Insulin release independent of a rise in cytosolic free $\mathrm{Ca}^{2+}$ by forskolin and phorbol ester. FEBS Lett 183: 430-432, 1985.

23. Park HY, Russakovsky V, Ohno S and Gilchrest BA: The beta isoform of protein kinase $\mathrm{C}$ stimulates human melanogenesis by activating tyrosinase in pigment cells. J Biol Chem 268: 11742-11749, 1993

24. Sasaki M, Horikoshi T, Uchiwa H and Miyachi Y: Up-regulation of tyrosinase gene by nitric oxide in human melanocytes. Pigment Cell Res 13: 248-252, 2000.

25. Kim DS, Park SH, Kwon SB, Li K, Youn SW and Park KC: (-)-Epigallocatechin-3-gallate and hinokitiol reduce melanin synthesis via decreased MITF production. Arch Pharm Res 27: 334-339, 2004.

26. Koo JH, Kim HT, Yoon HY, et al: Effect of xanthohumol on melanogenesis in B16 melanoma cells. Exp Mol Med 40: 313-319, 2008.

27. Lv N, Koo JH, Yoon HY, et al: Effect of Angelica gigas extract on melanogenesis in B16 melanoma cells. Int J Mol Med 20: 763-767, 2007.

28. Sharma B, Salunke R, Srivastava S, Majumder C and Roy P: Effects of guggulsterone isolated from Commiphora mukul in high fat diet induced diabetic rats. Food Chem Toxicol 47: 2631-2639, 2009.

29. Sarfaraz S, Siddiqui IA, Syed DN, Afaq F and Mukhtar H: Guggulsterone modulates MAPK and NF- $\kappa \mathrm{B}$ pathways and inhibits skin tumorigenesis in SENCAR mice. Carcinogenesis 29: 2011-2018, 2008 . 